\title{
Dynamics of solid growth under a gravitational field: Influence of the formation of a diffusive layer
}

\author{
Marcos F. Castez, Bárbara Blum, and Roberto C. Salvarezza \\ Instituto de Investigaciones Fisicoquímicas Teóricas y Aplicadas (INIFTA), UNLP, CONICET, Casilla de Correo 16, Sucursal 4, \\ (1900) La Plata, Argentina \\ Hernán G. Solari \\ Departamento de Física, Facultad de Ciencias Exactas y Naturales, Universidad de Buenos Aires, 1428 Buenos Aires, Argentina
}

(Received 3 January 2003; published 30 June 2003)

\begin{abstract}
We discuss the gravitational sedimentation of particles in terms of a stochastic model considering, in view of experimental evidence, that the aggregation to the growing surface (deposit) is mediated by the formation of a layer of suspended particles subject to gravitational forces, thermal agitation, as well as aggregation (contact) forces. The aggregation of such partially buoyant particles is ruled by the rates of occurrence of the different stochastic events: incorporation to the layer of suspended particles, sedimentation, and gravitationally biased diffusion. The model introduces bridges across different standard solid on solid deposition models which can be considered as limit cases of the present one. Analytical and numerical results show that for finite (realistic) deposits there are different regimes of aggregation including situations in which the deposit is grown completely during the transient time of the system.
\end{abstract}

DOI: 10.1103/PhysRevE.67.061605

PACS number(s): 81.10.Aj, 81.15.Aa, 05.10.Ln

\section{INTRODUCTION}

The growth of solids by sedimentation of particles has practical and theoretical relevance. From a theoretical point of view the mechanism of gravitational deposition of particles ranks among the simplest forms of growing a solid in a controlled situation and as such it represents a test bench for different solid on solid (SOS) $[1,2]$ deposition models and their analysis.

From a technological point of view, the preparation of "supercrystals" [3] by aggregation of nearly monodispersed nanoparticles or microparticles has attracted considerable interest. Crystals formed by the aggregation of $\mathrm{CdS}, \mathrm{Cu}_{2} \mathrm{Se}$, and $\mathrm{Fe}_{2} \mathrm{O}_{3}$ nanospheres with appealing optical and electrical properties have been prepared and characterized. However, synthetic opals constructed by sedimentation of submicron silica, $\mathrm{SiO}_{2}$, particles remain the most popular and paradigmatic supercrystals.

Particles in supercrystals are held together by van der Waals forces. Silica self-assembling to produce good quality opals requires small rates of particle aggregation which are close to the thermodynamical equilibrium. In such situations, aggregation does not happen unavoidably upon contact of the particles with the growing interface. In practical terms, the growth of these opals requires several days and proceeds with the formation of a "white clue," a diffusive layer of particles close to the growing surface [4].

The interface dynamics of this system has been studied by atomic force microscopy imaging, and interpreted using dynamic scaling arguments. Experimental results show that the supercrystals become rougher with increased deposited height in a form that (when restricted to a proper spatial and temporal region) is compatible with the scaling relations determined for random incorporation followed by relaxation models, such as the Edwards-Wilkinson (EW) model [5]. This model accounts of the preferential growth of the surface at valleys and the inhibition of growth at peaks (transformed into evaporation when the average deposition rate is subtracted from the model). However, it cannot account for the diffusive layer which has no room in this class of settings.

In the present work we introduce an indirect deposition model in which particles are incorporated at random to a suspended layer of almost buoyant particles which in turn can stick to the surface or bounce (most likely) downhill along the surface.

The model is framed in the standard population dynamics setting, i.e., as a Markov system with density dependent transition probabilities [6] and, as such, it contrasts with standard SOS models $[7,8]$ since the certainty of deposition rules is completely avoided.

Furthermore, our aim is to understand the role of the diffusive layer in the growth of opals as well as to characterize different experimental situations that can potentially affect the properties of the supercrystal. We will constrain our study to finite-size and finite-time growth processes completely avoiding the (cumbersome) infinite-size and infinitetime singular (and noncommuting) limits.

A second and important purpose of this work is to improve the standard analysis of stochastic models of crystal growth which could be later applied to other real and more complex situations. In this sense, the discussion includes scaling laws but moves beyond them estimating the times at which crossovers from one scaling law to another will happen due to the prevalence of different (kinds of) events.

The paper is organized as follows: Section II introduces the stochastic model; Secs. III-V discuss simple limit cases, the main aspects of the dynamics, and numerical results, respectively, while Sec. VI presents the summary and concluding remarks. The Appendix presents exact solutions for the case where there is no sensitivity to the deposited topography.

\section{MODEL DESCRIPTION}

We consider particles in a fluid media moving towards a solid surface (the substrate) driven by the gravitational force. 
TABLE I. Stochastic events considered, their effect, and transition rates $\mathcal{A}=1 /\left[P_{s}+(1\right.$ $\left.\left.-P_{S}\right)\left(\exp \left[-K\left(h_{i+1}-h_{i}\right)\right]+\exp \left[-K\left(h_{i-1}-h_{i}\right)\right]\right)\right]$.

\begin{tabular}{lcc}
\hline \hline \multicolumn{1}{c}{ Event } & Effect & Transition rate \\
\hline Rain & $c_{i} \rightarrow c_{i}+1$ & $R$ \\
$R$ movement & $\left\{c_{i}, c_{i+1}\right\} \rightarrow\left\{c_{i}-1, c_{i+1}+1\right\}$ & $c_{i} \mathcal{A}\left(1-P_{s}\right) \exp \left[-K\left(h_{i+1}-h_{i}\right)\right]$ \\
$L$ movement & $\left\{c_{i}, c_{i-1}\right\} \rightarrow\left\{c_{i}-1, c_{i-1}+1\right\}$ & $c_{i} \mathcal{A}\left(1-P_{s}\right) \exp \left[-K\left(h_{i-1}-h_{i}\right)\right]$ \\
Deposition & $\left\{c_{i}, h_{i}\right\} \rightarrow\left\{c_{i}-1, h_{i}+1\right\}$ & $c_{i} \mathcal{A} P_{s}$ \\
\hline \hline
\end{tabular}

The particles are not immediately aggregated to the substrate; rather, they have a certain probability of remaining in the interface, diffusing preferably to the sites of (local) minimum of potential energy (valleys). Particles will eventually attach to the substrate in an irreversible way. At the time $t$, there will be a number $C(t)$ of particles that have come close to the substrate but still have not been deposited. We say that these particles form the diffusive layer.

We formulate a minimal model that resembles the observed physics in terms of a stochastic (Markov) system suitable for computer simulations but also intended to be amenable to non-numerical analysis. We will try to keep the number of free parameters in the model as small as possible to simplify as well as to clarify the analysis.

Let $i=1, \ldots, L$ label the $i$ th site in a one-dimensional lattice. Consider the stochastic variables: number of particles above the $i$ th site in the diffusive layer, $c_{i}$; number of particles deposited on the $i$ th site, $h_{i}$. $h_{i}$ is measured in lattice units. One lattice unit in our model is equivalent to our particle width.

The evolution of the populations $h_{i}, c_{i}$ responds to stochastic events which can be classified in two large groups: (a) incorporation to the diffusive layer (particle rain or just "rain") and (b) transitions.

(a) Particle rain events consist in the incorporation of a particle into the diffusive layer at a constant rate $R$ transforming $c_{i} \rightarrow c_{i}+1$, and leaving the remaining variables unchanged.

(b) Transition events in the diffusive layer alter the state of the system but not the number of particles being considered.

The transition events are of three different kinds: deposition at a site $i$, and particle movement in the diffusive layer from site $i$ either to the left or to the right.

The different kinds of events and their transition rates are summarized in the Table I and illustrated in Fig. 1.

Note that the total transition rate for the transition events is $c_{i}$, i.e., we assume that the likelihood of a transition event

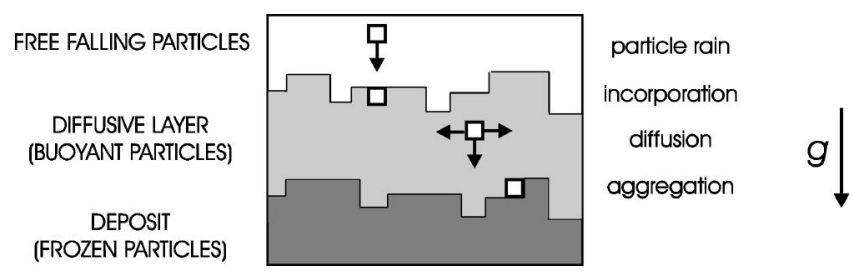

FIG. 1. Schematic illustration of the different events and their influence on the dynamics. at the site $i$ is proportional to the local concentration of particles in the diffusive layer.

The time between events is assumed to be exponentially distributed with density $\nu \exp (-\nu t)$, where $\nu=(R L+C)$ is a characteristic frequency and $C=\sum_{j}^{L} c_{i}$. This time distribution corresponds to the usual assumptions of population dynamics.

There are three free parameters in the model: $R, K$, and $P_{s}$. These parameters play the following role.

(a) $R$ is the rate at which particles are incorporated in the diffusive layer.

(b) We will show that the form in which the topography of the deposit influences the diffusive layer dynamics is controlled by $K$. For $K>0$ the particles are prone to accumulate in the regions corresponding to the valleys, for $K<0$ they tend to accumulate at the potential energy maxima (peaks), while for $K=0$ the diffusive layer dynamics is independent of the interface shape. Thus, $K$ is related to the competition between gravitational and thermal energies.

(c) The parameter $P_{s}$, normalized so that $0 \leqslant P_{s} \leqslant 1$, represents the probability that a particle sticks to the deposit once it impinges upon it. $P_{s}$ therefore is related to the relative occurrence of left or right movements within the diffusive layer in contrast with deposition events. As such, it controls the mean path traveled by the particles in the diffusive layer before attaching to the (frozen) substrate.

In particular, in the absence of gravitational effects ( $K$ $=0$ ), the particles perform an (unbiased) random walk that is interrupted by deposition events. In this case, the mean free path of a particle is directly related to the parameter $P_{S}$. Consider a particle incorporated into the diffusive layer, there are three possible events: deposit, and movement either to the left, or to the right. The latter are performed with probability

$$
p=\frac{2\left(1-P_{s}\right)}{2-P_{s}} .
$$

The probability for the particle to make $d$ steps in any direction before depositing is

$$
P_{d}=(1-p) p^{d}
$$

Since each step is an independent event, and the probability of moving either to the left or to the right are equal, the mean free path $\lambda$ is

$$
\lambda=\sqrt{\left\langle d^{2}\right\rangle}=\sqrt{p(p+1) /(1-p)^{2}} .
$$


The mean free path traveled by a particle in absence of gravitational effects is then given by

$$
\lambda=\sqrt{\frac{2\left(1-P_{s}\right)\left(4-3 P_{s}\right)}{P_{s}^{2}}},
$$

where distances are measured in terms of lattice units. We see that when $P_{s} \rightarrow 1$, we have $\lambda \rightarrow 0$, while for $P_{s} \rightarrow 0$, the mean distance traveled grows to infinity.

This picture does not remain valid if $K>0(K<0)$ since in such a case the particles tend to remain confined to the valleys (peaks), in which case $\lambda$ is expected to be related to the distance between valleys (peaks).

Part of the discussion of results will refer to the roughness of the deposit, a quantity of relevance in experiments and applications that consequently has received much theoretical attention. The roughness of the interface $W$ is defined as the root mean square of the deviations from the mean value of the site heights, i.e.,

$$
W^{2}(L, t)=\left\langle\left(h_{i}(t)-\left\langle h_{i}(t)\right\rangle_{L}\right)^{2}\right\rangle_{L},
$$

where the bracket \langle\rangle$_{L}$ denotes lattice average.

\section{ELEMENTARY ANALYSIS AND LIMIT CASES}

There are four regions in parameter space in which the present model is in correspondence with simpler models.

$P_{s}=1$ : There is no diffusion. The variables at each site are independent. At each site there is random deposition mediated by the suspended phase. Hence, the deposition process will be Poisson distributed (see the Appendix for an analytical deduction) and after a transient time it will behave as random deposition.

$K=0$ : The dynamics in the diffusive layer is not affected by the substrate topography. This implies that growth of roughness will be essentially the one that corresponds to the random deposition case, since the diffusive layer will average to a homogeneous layer. This case can be solved analytically (see the Appendix).

$K<0$ : There will be a tendency for particles to move towards local maxima where they will aggregate resulting in the formation of sharp peaks where the particles will accumulate, separated by regions where the diffusive layer is very thin (the probability of aggregating in the valleys is lower than at the peaks) and the deposits are negligible. In this case, the roughness increases quadratically with the total number of deposited particles [9].

$R \ll 1, K>1$ : In this case, the rain of particles is very slow in comparison with the aggregation process, hence, a rarefied diffusive layer is expected. For sufficiently large values of $K$, lateral movements in the diffusive layer will outrate the deposits, and the particle is expected to explore large regions of the surface being deposited with larger probability in those sites with (local) minimal gravitational energy. In Fig. 2, we compare the evolution of the roughness in a typical realization of the stochastic process with an essentially deterministic model ruled by a single specification: "Particles are initially deposited on a site (randomly chosen) and

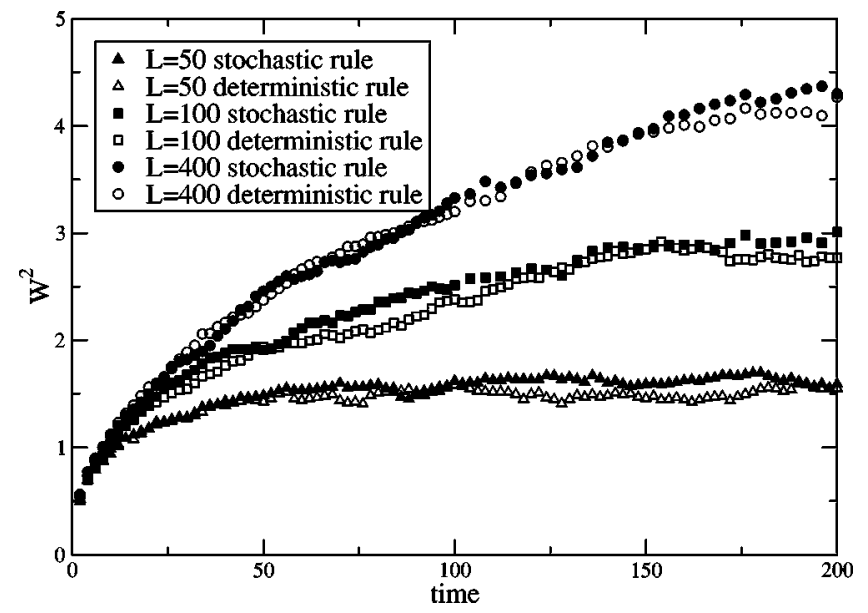

FIG. 2. Quadratic roughness as a function of time for our model (filled symbols) and for a deterministic model (hollow symbols) of deposition on semistable sites, for different lattice sizes. $P_{s}=0.95$, $R=10^{-5}$, and $K=6$.

travel across the surface until they find the first semistable (i.e., with $h[i] \leqslant h[i \pm 1]$ ) site where they are aggregated to the surface." The unit of time is chosen as that necessary to incorporate the equivalent to $1 \mathrm{ML}$ of particles to the system.

The scaling exponents of the adatom model are in good agreement with those from other random deposition models based on surface relaxation [1], as well as those found for the EW equation [5]. While much theoretical work has been performed pertaining to deposition models, related experiments concerning deposition of colloidal suspensions are few, to our knowledge, and information relevant to our model even scarcer. Xin-Ya Lei et al. [10] investigate polymer deposition in a $(1+1)$-dimensional system - they only analyze results in the final, apparently stationary, regime. Salvarezza et al. report, in $(2+1)$-dimensional experiments, scaling exponents consistent with the EW equation. Their roughness is measured as a function of average deposit thickness $\langle h\rangle$, but not of time.

In the remainder of this work, we will focus our analysis on the implications of the existence of a diffusive layer, without limiting our analysis to asymptotic properties. We have no knowledge of experiments performed in this general case. The aim of what follows is therefore to allow experiments to be planned in the more frequently achieved transient regime.

\section{THE DIFFUSIVE LAYER AND DEPOSIT KINETICS}

Particles are constantly incorporated to the diffusive layer as a result of the particle rain, while at the same time the diffusive layer is depleted by the deposition process. Being the aggregation rate proportional to the number of particles in the diffusive layer, we can expect that, on an average, a balanced situation is reached.

If $C(t)$ stands for the total number of particles in the diffusive layer, the expected average number at a site will be $c(t)=C(t) / L$. The average number of aggregated particles will be called $h(t)$.

In the $K=0$ case, the average width of the diffusive layer 


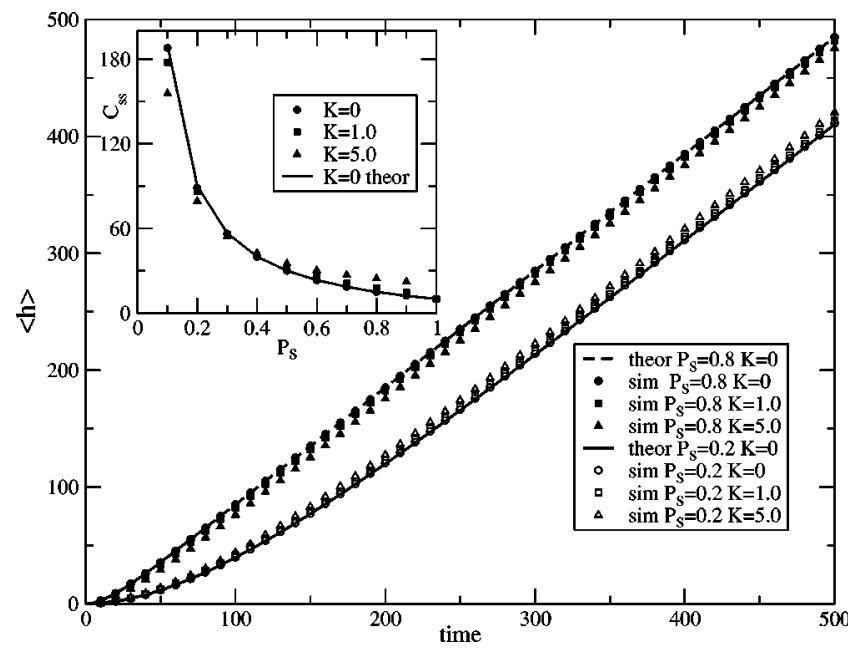

FIG. 3. Evolution of the mean substrate thickness as a function of time: analytical result $(K=0)$, and simulations for $P_{s}=0.2$ and $P_{s}=0.8$ with different $K$ values. $L=100, R=10$ for an average over 100 independent runs. The time unit corresponds to the incorporation of $1 \mathrm{ML}$ of particles. Inset: Steady state concentration as a function of $P_{s}$ for different $K$.

in the steady state is $c_{s s}=R\left(2-P_{s}\right) / P_{s}$ (A15), while the average deposit height evolves as

$$
\langle h\rangle=R t-c_{s s}\left(1-e^{-\left[P_{s} /(2-P s)\right] t}\right),
$$

assuming an initial condition corresponding to a homogeneous state $[c(0)=h(0)=0]$.

Note that for $t \gg\left(2-P_{s}\right) / P_{s}$, the mean height of the deposit grows linearly with time, $h \sim t$, a relation that is assumed in most models studied in the literature. However, when $t \leqslant\left(2-P_{s}\right) / P_{s}$, the average height grows as $h \sim t^{2}$.

In Fig. 3, we present results corresponding to Monte Carlo simulations for the case $K \neq 0$. Two families of curves are shown. The first one corresponds to $P_{s}=0.8$, while the second family corresponds to $P_{s}=0.2$. We can verify that for the family with $P_{s}=0.8$, the average deposit decreases with $K$ for any fixed time, while on the contrary, for $P_{s}=0.2$, the deposited height increases with $K$ for any fixed time. We can see that even after a deposit of $500 \mathrm{ML}$ (in real situations, a width in the micrometer scale) expression (6) represents a good approximation to the evolution of the deposit, even for large values of $K$.

The result suggests that when the diffusion of particles is slow $\left(P_{s} \approx 1\right)$, the diffusive layer is rarefied in the valleys, delaying the deposition process, while at the same time deposition at the peaks is increasingly inhibited by increasing the values of $K$.

When $P_{s} \approx 0$, most of the particles are available for deposition at the valleys and the effect of increasing $K$ is to increase the effective deposit rate.

Evidently, there is a value of $P_{s}$ for which the situation is intermediate between both extreme cases. The situation is shown in the inset of Fig. 3. For $P_{s} \sim 0.3$, it can be seen that the concentration of the steady state is practically independent of $K$.

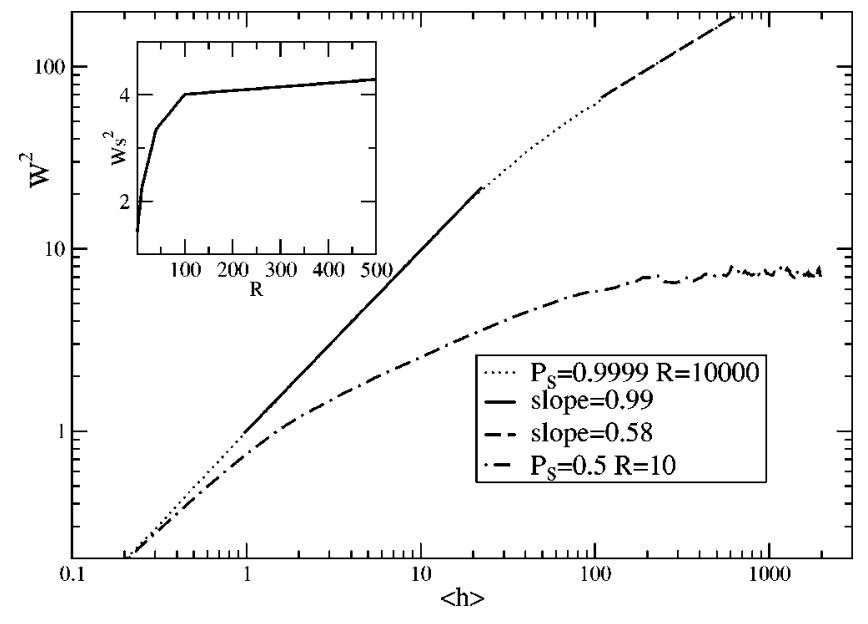

FIG. 4. Quadratic roughness vs mean deposit thickness, for two extreme values of diffusive layer width. $L=100, K=0.5$, average over 100 independent runs. Inset: Growth of the deposit roughness vs rain rate.

\section{NUMERICAL RESULTS}

\section{A. Roughness at saturation}

In this section, we show how both the characteristic scales associated with the different power law regimes, and their crossovers, are affected by the presence of a diffusive layer, meaning that, under certain circumstances that could be met in experimental work, an adequate estimation of the width of the diffusive layer might be more relevant to nanotechnological processes than the universal exponents in the asymptotic regime.

The basic process that introduces smoothness into the deposit consists in the accumulation of particles in correspondence with the valleys, and the rarefaction of the diffusive layer in correspondence with the peaks of the deposit, as a result of a biased diffusion process. The smoothing process enters in competition with the intrinsic fluctuations due to the rain process. We estimate the size of these fluctuations to be of the order of $c^{1 / 2}$, with $c$ the mean width of the diffusive layer.

We further expect that in the limit case in which $c$ is very large, the biased diffusion will result inefficient to compensate the size of the fluctuations. Because of this, we expect the evolution of roughness to be affected by the width of the diffusive layer. Evidence of this effect can be found in Fig. 4 where we can see that with a very thick diffusive layer the random-deposition regime survives even after a deposit of 20 or $30 \mathrm{ML}$, in contrast to the situation where the diffusive layer is thin and the random-deposition regime ends soon after the first monolayer is deposited.

The saturation value for the roughness of the interface as a function of the rain rate is shown in Fig. 4 (inset). As a result of the balance between smoothing and fluctuations described, the saturation values of the roughness will depend on the rain rate: the slower the particle rain, the better the film quality, a conclusion that seems to be intuitive for sedimentation models but has not been accounted for by other models and/or studies. 


\section{B. Influence of the diffusive layer on the characteristic exponents}

In most of the models studied in the literature $[7,8]$ it is found that the roughness satisfies the dynamic scaling hypotheses [11] implying that the dependence of $W^{2}$ on time is of the form

$$
W^{2}(L, t)= \begin{cases}\sim t & \text { if } t<t_{0} \\ \sim t^{2 \beta} & \text { if } t_{0}<t<t_{1}(L) \\ \text { const } & \text { if } t_{1}(L)<t,\end{cases}
$$

where $\beta$ is a characteristic exponent of the system. It is expected that characteristic exponents be invariant in front of changes in the rules that define the model, as long as the basic underlying physics is preserved.

However, the fact that in our system the deposit does not (initially) grow linearly with time, Fig. 5, implies that the graph of $W^{2}$ against time is not in agreement with the standard picture.

The initial transient time $t_{0}$ roughly corresponds to the time for depositing $1 \mathrm{ML}$ and is characterized by the absence of correlations among sites. The evolution of the roughness is then expected to match that corresponding to random deposition, i.e., $W^{2}(L, t) \propto t$.

However, the presence of a diffusive layer, introduces a characteristic time scale $t_{k}$ that corresponds to the time required to reach the steady state concentration. From Eq. (6), we can estimate this time as $t_{k}=\left(2-P_{s}\right) / P_{s}$. The observable exponents corresponding to $W^{2}$ vs $t$ associated with the different regimes will depend on the value of $t_{k}$ compared to the other time scales present such as $t_{0}$ (deposit of $1 \mathrm{ML}$ ) and $t_{1}$, the saturation time of the deposit in terms of the roughness. This means that different scaling laws will emerge from the $W^{2}$ vs $t$ plots depending on whether $t_{k}<t_{0}, t_{0}<t_{k}<t_{1}$, or $t_{1}<t_{k}$.

In particular, it can be seen (Fig. 5) that for times shorter than $t_{k}$ (the situation in which $h \sim t^{2}$ ), and $t_{0}<t_{k}<t_{1}$, the observed exponents in the $W^{2}$ vs $t$ curves are (approximately) doubled with respect to those observed in the $W^{2}$ vs $h$ curves.

\section{SUMMARY AND CONCLUDING REMARKS}

We have introduced a simple model for sedimentation where only gravitational forces and diffusion effects are included. The model describes both the particle aggregation and a layer of buoyant particles that we have named the diffusive layer.

The model introduced follows the standard formulation of population dynamics and as such can be analyzed. In this context, it is important to notice that most results will be in terms of statistical estimators, for example, the asymptotic value of the mean thickness of the diffusive layer can take any real value. An average number of $10^{-4}$ particles can only be interpreted as a buoyant particle every $10^{4}$ sites, i.e., a lonely particle trying to find the proper place where to attach to the surface.

By changing parameters, the model can bridge between
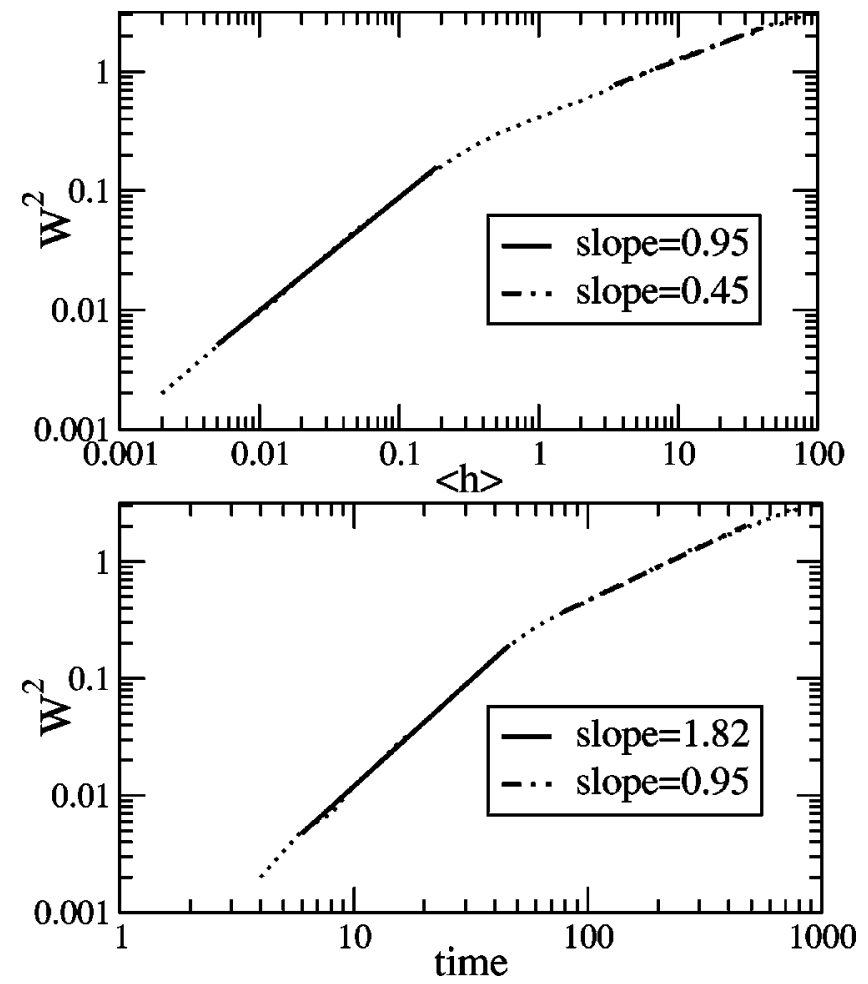

FIG. 5. Quadratic roughness as a function of the mean height (upper) and as a function of time (lower) in the $h \sim t^{2}$ region, showing the (approximate) doubling of the scaling exponents. $L=100$, $P_{s}=0.2, R=500, K=5$, average over 100 independent runs. The time unit corresponds to the incorporation of $1 \mathrm{ML}$ of particles.

situations which are observationally different as, for example, those situations where a white clue is formed, and those of extremely rarefied diffusive layers which cannot be directly observed.

The present work focuses in growing thin layers and as such the question of the "universality class" of the model lies beyond the scope and possibilities of the present study. Nevertheless, observed values of critical exponents computed during the simulations are compatible with the "Edwards-Wilkinson" class. It is worth noticing that the time required by the system to abandon the "randomdeposition" transient strongly depends on the thickness of the diffusive layer.

Among the important features of the present model and its mathematical study, we emphasize on the following points.

(a) There is a single model for several different situations. Differences are managed through continuous changes in parameter values rather than in sharp changes of rules.

(b) The limit case where there is no sensitivity to the deposit topography can be solved in exact form, opening the possibility of applying (developing) perturbation theory to cases with weak dependence on the deposited profile.

(c) The model at no point assumes a linear relation between time and average deposited height, and predicts a slower buildup of the deposit at the beginning of the deposition process. Furthermore, for any given deposit thickness it is possible to make the full deposition process in the transitory regime. 
(d) It is possible to estimate, within the approximate model, times for the prevalence of one or another class of events. The prevalence of different events will be apparent in the rate of change of different statistical estimators as, for example, crossover of scaling exponents.

(e) The model predicts a dependence of the final smoothness with the particle incorporation rate not present in previous models.

(f) The model predicts a complete statistical equivalence of the deposit (as a function of time) in all the cases where no sensitivity to the topography of the deposit is present and the particle rain is homogeneous. This fact suggests that experiments trying to probe the underlying physics might benefit from an inhomogeneous rain of particles which is definitely not recommended if a smooth homogeneous surface is the final objective.

\section{ACKNOWLEDGMENTS}

B.B., R.C.S., and H.G.S. are supported by the Carrera del Investigador Científico del CONICET, Argentina. This work has been performed as part of the project PICT 99-6-5030 of FONCYT (Argentina). M.F.C. acknowledges CONICET (Argentina) for financial support.

\section{APPENDIX: ANALYTICAL SOLUTION FOR $K=0$}

In this section, we explore analytical solutions for the case $K=0$ (no sensitivity to the topography of the deposit).

\section{Derivation of the probability generating function}

The stochastic variables are the concentration at the different sites, $c_{i}$ with $i=1, \ldots, L$, and the number of deposited particles $h_{i}$ on each site.

The master equation reads

$$
\begin{aligned}
\dot{P}(\mathbf{c}, \mathbf{h}, t)= & \sum_{i=1}^{L}\left[R P\left(c_{i}-1\right)+B\left(c_{i}+1\right) P\left(c_{i-1}-1, c_{i}+1\right)\right. \\
& +B\left(c_{i}+1\right) P\left(c_{i}+1, c_{i+1}-1\right)+A\left(c_{i}+1\right) P\left(c_{i}\right. \\
& \left.\left.+1, h_{i}-1\right)\right]-\left[L R+(2 B+A) \sum_{i=1}^{L} c_{i}\right] P
\end{aligned}
$$

where

$$
B=\frac{1-P_{s}}{2-P_{s}} \quad \text { and } \quad A=\frac{P_{s}}{2-P_{s}}
$$

On the right side of Eq. (A1), we have highlighted the notation including only the arguments of the probabilities $P$ that change with the event associated to the term.

The generating function is defined by [12]

$$
\Psi(\mathbf{q}, \mathbf{s}, t)=\sum_{\{\mathbf{c}, \mathbf{h}\}} q_{1}^{c_{1}} \cdots q_{L}^{c_{L}} s_{1}^{h_{1}} \cdots s_{L}^{h_{L}} P(\mathbf{c}, \mathbf{h}, t)
$$

and satisfies the partial differential equation:

$$
\begin{aligned}
\dot{\Psi}= & R\left(\sum_{n} q_{n}-L\right) \Psi+\sum_{n}\left[B\left(q_{n-1}+q_{n+1}\right)\right. \\
& \left.-(2 B+A) q_{n}+A s_{n}\right] \frac{d \Psi}{d q_{n}} .
\end{aligned}
$$

Let $\Psi=e^{\Phi}$. The corresponding equation for $\Phi$ reads

$$
\begin{aligned}
\dot{\Phi}= & R\left(\sum_{n} q_{n}-L\right)+\sum_{n}\left[B\left(q_{n-1}+q_{n+1}\right)\right. \\
& \left.-(2 B+A) q_{n}+A s_{n}\right] \frac{d \Phi}{d q_{n}} .
\end{aligned}
$$

Since (A4) is a quasilinear equation, its solutions can be obtained by the method of characteristics [13].

The system of characteristic equations associated to Eq. (A4) are

$$
\begin{aligned}
\frac{d t}{1} & =\ldots=\frac{d q_{i}}{(2 B+A) q_{i}-B q_{i-1}-B q_{i+1}-A s_{i}} \\
& =\cdots=\frac{d \Phi}{R \sum_{n} q_{n}-L R} .
\end{aligned}
$$

After integrating Eq. (A5), we get

$$
\Phi=\frac{R}{A} Q+\frac{R}{A}(S-L) \ln |Q-S|+G\left(v_{1}, \ldots, v_{L}\right),
$$

where $Q=\Sigma q_{i}, S=\Sigma s_{i}, G$ is a function to be determined using the initial conditions, and $v_{i}$ are the integral surfaces corresponding to the $L$ characteristic equations, i.e., $v_{j}\left(q_{i}(t), t\right)=$ const $_{j}$ when $q_{i}(t)$ satisfies

$$
\dot{q_{i}}=B \sum_{j} M_{i j} q_{j}+A\left(q_{i}-s_{i}\right)
$$

Here, $M_{i j}$ are the elements of an $L^{2}$ matrix defined as

$$
M_{i j}=2 \delta_{i j}-\delta_{i(j-1 \bmod L)}-\delta_{i(j+1 \bmod L)},
$$

$M$ is symmetric and non-negative matrix, with eigenvalues

$$
\lambda_{k}=2\left[1-\cos \left(\frac{2 \pi k}{L}\right)\right]
$$

each one with degeneration two. The corresponding eigenvectors read

$$
T_{n j}=\exp \left(i \frac{2 \pi}{L}(n-1)(j-1)\right),
$$

with $k=1,2, \ldots, L$ (there is an arbitrary election within the degenerated subspaces).

Equation (A7) is solved using standard methods ("variation of the constants, for example") arriving in this form to the general solution of Eq. (A4) that reads 


$$
\begin{aligned}
\Phi(t)= & \frac{R}{A} Q+\frac{R}{A}(S-L) \ln |Q-S| \\
& +G\left(e^{-\left(A+\lambda_{i} B\right) t} \sum_{j} T_{i j}\left(q_{j}-c_{j}\right)\right),
\end{aligned}
$$

where $c_{i}$ satisfies $\Sigma_{j}\left(B M_{i j}+A \delta_{i j}\right) c_{j}=A s_{i}$.

Considering the initial condition that corresponds to the certainty of having $c_{i}^{0}$ particles in the site $i$ of the diffusive layer, and $h_{i}^{0}$ particles deposited in the site $i$,

$$
\Phi(0)=\sum_{n}\left(c_{n}^{0} \ln q_{n}+h_{n}^{0} \ln s_{n}\right),
$$

we obtain the expression of $G$ for this family of initial conditions

$$
\begin{aligned}
G\left(v_{1}, v_{2}, \ldots, v_{L}\right)= & \sum_{k}\left(c _ { k } ^ { 0 } \operatorname { l n } \left\{\sum _ { n } \left(\frac { 1 } { L } \operatorname { e x p } \left[-i \frac{2 \pi}{L}(k-1)\right.\right.\right.\right. \\
& \left.\left.\left.\times(n-1)] v_{n}\right)+c_{k}\right\}+h_{k}^{0} \ln s_{k}\right) \\
& -\frac{R}{A}\left(v_{1}+S\right)-\frac{R}{A}(S-L) \ln \left|v_{1}\right| .
\end{aligned}
$$

Finally, the generating function with the initial configuration $c_{k}^{0}, h_{k}^{0}$ is

$$
\begin{aligned}
\Psi(\mathbf{q}, \mathbf{s}, t)= & \exp \left(\frac{R}{A}(Q-S)\left(1-e^{-A t}\right)+R t(S-L)\right) \\
& \times \prod_{k=1}^{L}\left(\sum_{n j} \frac{1}{L} e^{i(2 \pi / L)(n-1)(j-k)}\right. \\
& \times e^{-\left(A+\lambda_{n} B\right) t} q_{j}+\sum_{n j} \frac{A}{L} e^{i(2 \pi / L)(n-1)(j-k)} \\
& \left.\times \frac{\left(1-e^{-\left(A+\lambda_{n} B\right) t}\right)}{\left(A+B \lambda_{n}\right)} s_{j}\right)^{c_{k}^{0}}\left(s_{k}\right)^{h_{k}^{0}} .
\end{aligned}
$$

\section{Particular cases}

We shall briefly explore the meaning of the results obtained in the preceding section considering the following cases.

(a) Initially there is no particle in the system. $c_{i}^{0}=0, h_{i}^{0}$ $=0$.

In this case the marginal distributions $f(c)$ and $g(h)$ are Poisson-like with mean values given by

[1] F. Family, J. Phys. A 19, L441 (1986).

[2] M.R. Wilby, D.D. Vvedensky, and A. Zangwill, Phys. Rev. B 46, 12896 (1992).

[3] G.L. Timp, Nanotechnology (Springer-Verlag, New York,

$$
\begin{gathered}
\langle c\rangle=\sigma_{c}^{2}=\frac{R}{A}\left(1-e^{-A t}\right), \\
\langle h\rangle=\sigma_{h}^{2}=R t-\frac{R}{A}\left(1-e^{-A t}\right) .
\end{gathered}
$$

(b) No diffusion case: $P_{S}=1$.

In this case, $B=0$ and $A=1$. The generating function is then

$$
\begin{aligned}
\Psi_{\left(P_{s}=1\right)}= & \prod_{k=1}^{L} \exp \left[R\left(q_{k}-s_{k}\right)\left(1-e^{-t}\right)\right. \\
& \left.+R t\left(s_{k}-L\right)\right] s_{k}^{h_{k}^{0}}\left[e^{-t}\left(q_{k}-s_{k}\right)+s_{k}\right]^{c_{k}^{0}},
\end{aligned}
$$

which factorizes with respect to the site variables as a manifestation that the sites become statistically independent when there is no diffusion.

(c) Asymptotic limit $t \rightarrow \infty$.

The marginal distributions in the $t \rightarrow \infty$ limit are

$$
\begin{aligned}
& f_{\text {asympt }}\left(q_{n}\right)=\exp \left(\frac{R}{A}\left(q_{n}-1\right)\right), \\
& g_{\text {asympt }}\left(s_{n}\right)= \exp \left[\operatorname{Rt}\left(s_{n}-1\right)\left(1-\frac{R}{A}\right)\right]\left(s_{n}\right)_{n}^{h_{n}^{0}} \\
& \times \prod_{k}\left[\frac{1}{L} \sum_{j}\left(\frac{\exp \left[i \frac{2 \pi}{L}(j-1)(n-k)\right]}{1+\frac{B}{A} \lambda_{j}}\right)\right. \\
&\left.\times\left(s_{n}-1\right)+1\right] .
\end{aligned}
$$

We see that the diffusive layer variables evolve to the stationary state distribution losing "memory" of the initial state. In contrast, the marginal generating function for the deposited particles, $g_{\text {asympt }}\left(s_{n}\right)$, is still dependent on the initial conditions.

Roughly speaking, we can say that, for very large times, the initial condition will only be reflected in the statistical properties of the deposited layer, and not in those of the diffusive layer.
1999).

[4] R.C. Salvarezza et al., Phys. Rev. Lett. 77, 4572 (1996).

[5] S.F. Edwards and D.R. Wilkinson, Proc. R. Soc. London, Ser. A 381, 17 (1982). 
[6] S.N. Ethier and T.G. Kurtz, Markov Processes (Wiley, New York, 1986).

[7] A.L. Barabasi and H.E. Stanley, Fractal Concepts in Surface Growth (Cambridge University Press, Cambridge, 1995).

[8] P. Meakin, Fractals, Scaling and Growth Far from Equilibrium (Cambridge University Press, Cambridge, 1998).

[9] M.F. Castez (unpublished).
[10] C.-H.Z. Xin-Ya Lei, Peng Wan, and N.-B. Ming, Phys. Rev. E 54, 5298 (1996).

[11] F. Family and T. Vicsek, J. Phys. A 18, L75 (1985).

[12] N.G.V. Kampen, Stochastic Processes in Physics and Chemistry (North-Holland, Amsterdam, 1981).

[13] I.N. Sneddon, Elements of Partial Differential Equations (McGraw-Hill, New York, 1957). 